\title{
Fine Fuzzy sp Closed Sets in Fine Fuzzy Topological Spaces
}

\author{
R. Nandhini, D. Amsaveni
}

\begin{abstract}
The main view of this article is the extended version of the fine topological space to the novel kind of space say fine fuzzy topological space which is developed by the notion called collection of quasi coincident of fuzzy sets. In this connection, fine fuzzy spclosed sets are introduced and studied some features on it. Further, the relationship between fine fuzzy spclosed sets with certain types of fine fuzzy closed sets are investigated and their converses need not be true are elucidated with necessary examples. Fine fuzzy spcontinuous function is defined as the inverse image of fine fuzzy closed set is fine fuzzy spclosed and its interrelations with other types of fine fuzzy continuous functions are obtained. The reverse implication need not be true is proven with examples. Finally, the applications of fine fuzzy spcontinuous function are explained by using the composition.
\end{abstract}

Keywords: Fine fuzzy topological space, Fine fuzzy spclosed set, Fine fuzzy continuous, Fine fuzzy $\mathfrak{s p}^{* *}$ continuous. Subject Classification Primary: 54A05, 54A10, 54A20.

\section{INTRODUCTION}

The Fuzzy set theory uses the linguistic variable to represents imprecise concepts in many real-life applications in engineering, robotics, spacial objects, biosciences, etc. It is a marvelous tool for modeling in the various kinds of uncertainty associated with imprecision and vagueness. American Cybernatist Lofti A. Zadeh [12] initiated the theory of fuzzy set in 1965. Later, in the year 1968, the Chang [3] extended the notion of a fuzzy topology. Further, Power P. L. and Rajak K[9] investigated fine topological space, was particular case of generalized topological space. The views on sp-open sets in general topology and fuzzy sp-open sets were introduced by Dontcev, Przemski in 1996 [5] and Hakeem A. Othman [6] in 2011 respectively. This paper is mainly focused to the extension of fine topological space. The approach on fine fuzzy $\mathfrak{s p}$ closed sets in fine fuzzy topological spaces were well developed and studied. Further, the interrelations of fine fuzzy $\mathfrak{s p}$ closed sets with distinctive types of fine fuzzy

Revised Manuscript Received on February 05, 2020.

* Correspondence Author

R. Nandhini*, Department of Mathematics, Sri Sarada College for Women(Autonomous), Salem, India, $\quad$ E-mail: nandhinirajamradha@gmail.com

D. Amsaveni, Department of Mathematics, Sri Sarada College for Women (Autonomous), Salem, India, E-mail: d_ amsaveni@rediffmail.com

(C) The Authors. Published by Blue Eyes Intelligence Engineering and Sciences Publication (BEIESP). This is an open access article under the CC BY-NC-ND license (http://creativecommons.org/licenses/by-nc-nd/4.0/) closed set were investigated. Later, we defined the fine fuzzy $\mathfrak{s p}$ continuous functions and briefly discussed on its properties.

\section{PRELIMINARIES}

\section{Definition 2.1[11]}

Let $\mathrm{X}$ be a space of points and I be the unit interval $[0,1]$. A fuzzy set $\lambda$ in $X$ is a mapping from $X \rightarrow I$

\section{Definition 2.2[3]}

A fuzzy topology is a family $\mathrm{T}$ of fuzzy sets in $\mathrm{X}$ which satisfies the following conditions

(i) $\quad 0$ and $1 \in \mathrm{T}$.

(ii) $\quad$ If $\mu, \delta \in \mathrm{T}$, then $\mu \wedge \delta \in \mathrm{T}$.

(iii) If $\mu_{i} \in \mathrm{T}$ for each $\mathrm{i} \in \mathrm{I}$ then $\mathrm{V} \mu_{i} \in \mathrm{T}$, then $\mathrm{T}$ is called a fuzzy topology and the pair $(\mathrm{X}, \mathrm{T})$ is a fuzzy topological space.

\section{Definition 2.3[3]}

Let $\mathrm{f}$ be a function from $\mathrm{X}$ to $\mathrm{Y}$. Let $\mathrm{B}$ be a fuzzy set in $\mathrm{Y}$ with membership function $\mu_{B}(y)$. Then the inverse of $\mathrm{B}$, written as $f^{-1}[B]$, is a fuzzy set in $\mathrm{X}$ whose membership function is defined by $\mu_{f^{-1}[B]}(x)=\mu_{B}(f(x))$ for all $\mathrm{x}$ in $\mathrm{X}$. Conversely, let $\mathrm{A}$ be a fuzzy set in $\mathrm{X}$ with membership function $\mu_{A}(x)$. The image of $\mathrm{A}$, written as $f[\mathrm{~A}]$, is a fuzzy set in $\mathrm{Y}$ whose membership function is given by $\mu_{f[A]}(y)=$

$$
\left\{\begin{array}{cc}
\text { sup } \\
z \in f^{-1}[y] \quad\left\{\mu_{A}(z)\right\} & \text { if } f^{-1}[y] \text { is non empty } \\
0 & \text { other wise. }
\end{array}\right.
$$

for all $\mathrm{y}$ in $\mathrm{Y}$, where $f^{-1}[y]=\{x \backslash f(x)=y\}$.

\section{Definition 2.4[6]}

A fuzzy subset $u$ of fuzzy space $X$ is called fuzzy sp-open (fuzzy sp-closed) set if $\mathrm{u} \leq$ Int $\mathrm{cl} \mathrm{u} \vee \mathrm{cl}$ Int $\mathrm{u}$ ( Int cl u $\wedge \mathrm{cl}$ Int $\mathrm{u} \leq \mathrm{u}$ ). The class of all fuzzy sp-open (fuzzy sp-closed) sets in X will be denoted by FSP-O(X) (FSP-C(X)).

\section{Definition 2.5[9]}

Let $(X, \tau)$ be a topological space and define $\tau\left(A_{\alpha}\right)=$ $\tau_{\alpha}($ say $)=\left\{G_{\alpha}(\neq \mathrm{X}): \quad G_{\alpha} \cap A_{\alpha} \neq \phi\right.$, for $A_{\alpha} \in \tau$ and $A_{\alpha} \neq \phi$, $X$, for some $\alpha \in J$, where $J$ is the index set $\}$. Define $\tau_{f}=$ $\left\{\phi, X, \bigcup_{\alpha \in \mathrm{J}}\left\{\mathrm{T}_{\alpha}\right\}\right\}$. The collection $\tau_{f}$ of subsets of $\mathrm{X}$ is called the fine collection of subsets of $X$ and $\left(X, \tau, \tau_{f}\right)$ is said to be the fine space $X$ generated by the topology $\tau$ on $X$. 


\section{FINE FUZZY $\mathfrak{s p ~ C L O S E D ~ S E T S ~ I N ~ F I N E ~ F U Z Z Y ~}$ TOPOLOGICAL SPACES}

\section{Definition 3.1}

Let us consider the fuzzy topological space $(\mathcal{X}, \mathcal{T})$ and let $\mathcal{T}\left(\lambda_{\alpha}\right)=\mathcal{T}_{\alpha}=\left\{\mu_{\alpha}(\neq \mathrm{1}) \quad \mu_{\alpha} \wedge \lambda_{\alpha} \neq 0\right.$, for $\quad \lambda_{\alpha} \in$ $\mathcal{T}_{\alpha}$ and $\lambda_{\alpha} \neq 0,1$,

Where $\mathrm{J}$ is the indexed set for some $\alpha \in \mathrm{J}\}$. Then the collection $\mathcal{T}_{f}=\left\{0_{\mathrm{X}}, 1_{\mathrm{X}}, \bigcup_{\alpha \in \mathrm{J}}\left\{\mathrm{T}_{\alpha}\right\}\right\}$ is said to be fine fuzzy topology on $\mathcal{X}$ and $\left(\mathcal{X}, \mathcal{T}, \mathcal{T}_{f}\right)$ is the fine fuzzy topological space. It is denoted by $\mathcal{F} f T S$.

\section{Definition 3.2}

A fuzzy subset $\lambda$ of a $\mathcal{F} f T S$, is fine fuzzy open set of $\mathcal{X}(F f O(X))$, if $\lambda \in \mathcal{T}_{f}$ and its complement is denoted by fine fuzzy closed set $(F f C(X))$ of $\mathcal{X}$.

\section{Definition 3.3}

Let $\mu \in I^{x}$. Then the fine fuzzy interior of $\mu$ is denoted and defined by

$\operatorname{FfInt}(\mu)=\vee\{v: \mu \geq v, \mu$ is a fine fuzzy open set of $\mathcal{X}\}$

\section{Definition 3.4}

Let $v \in I^{x}$. Then the fine fuzzy closure of $v$ is denoted and defined by $\operatorname{FfCl}(v)=\wedge\{\mu: v \leq \mu, v$ is a fine fuzzy closed set of $\mathcal{X}\}$

\section{Definition 3.5}

Let $\psi:\left(\mathcal{X}, \mathcal{T}, \mathcal{T}_{f}\right) \rightarrow\left(\mathcal{Y}, \sigma, \sigma_{f}\right)$ be a mapping if $\psi^{-1}(\lambda) \in F f O(X)$ for each fine fuzzy open set $\lambda$ of $\mathcal{y}$, then $\psi$ is called fine fuzzy- irresolute.

\section{Definition 3.6}

A fine fuzzy subset $\lambda$ of $\mathcal{X}$ is called

1. fine fuzzy pre closed $(F f p C)$ if $\operatorname{FfCl}(\operatorname{FfInt}(\lambda)) \leq \lambda$

2. fine fuzzy semi closed $(F f s C)$ if $\operatorname{FfInt}(\operatorname{FfCl}(\lambda)) \leq \lambda$

3. fine fuzzy $\alpha$-closed $(F f \alpha C)$ if $\operatorname{FfCl}(\operatorname{FfInt}(\operatorname{fFCl}(\lambda))) \leq \lambda$.

4. fine fuzzy $\beta$-closed $(F f \beta C)$ $\operatorname{FfInt}(\operatorname{FfCl}(\operatorname{FfInt}(\lambda))) \leq \lambda$.

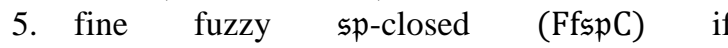
$\operatorname{FfCl}(\operatorname{FfInt}(\lambda)) \wedge \operatorname{FfInt}(\operatorname{FfCl}(\lambda)) \leq \lambda$.

The class of all fine fuzzy $\mathfrak{s p}$ closed set in $X$ is denoted by $\operatorname{Ff} \mathfrak{s p C}(\mathcal{X})$ and its complement is fine fuzzy $\mathfrak{s p}$ open set is $\operatorname{Ff} \mathfrak{s p O}(\mathcal{X})$ similarly for all the set

\section{Definition 3.7} fuzzy set of $\chi$. Then,

Let $\left(\mathcal{X}, \mathcal{T}, \mathcal{T}_{f}\right)$ be a $\mathcal{F} f T S$. Let $\lambda$ be fine

1. $\operatorname{FfpInt}(\mu)=\mathrm{v}\{\mu: \mu \leq v, \mu \in \operatorname{Ffp} O(\mathcal{X})\} \quad$ is fine fuzzy pre Interior.

2. $\operatorname{FfpCl}(v)=\wedge\{v: v \geq \mu, v \in F f p C(\mathcal{X})\}$ is fine fuzzy pre closure.

3. $F f \operatorname{sint}(\mu)=\vee\{\mu: \mu \leq v, \mu \in F f s O(\mathcal{X})\}$ is fine fuzzy semi Interior.
4. $\operatorname{Ffscl}(v)=\wedge\{v: v \geq \mu, v \in F f s C(\mathcal{X})\}$ is fine fuzzy semi closure.

5. $\operatorname{Ff} \alpha \operatorname{Int}(\mu)=\vee\{\mu: \mu \leq v, \mu \in F f \alpha O(\mathcal{X})\}$ is fine fuzzy $\alpha$-Interior.

6. Ff $\alpha C l(v)=\wedge\{v: v \geq \mu, v \in F f \alpha C(X)\}$ is fine fuzzy $\alpha$-closure.

7. $\operatorname{Ff} \beta \operatorname{Int}(\mu)=\vee\{\mu: \mu \leq v, \mu \in \operatorname{Ff} \beta O(\mathcal{X})\}$ is fine fuzzy $\beta$-Interior.

8. $\operatorname{Ff} \beta C l(v)=\wedge\{v: v \geq \mu, v \in F f \beta C(X)\}$ is fine fuzzy $\beta$-closure.

9. $\operatorname{Ff}_{\mathfrak{s p} \operatorname{Int}}(\mu)=\mathrm{V}\{\mu: \mu \leq \nu, \mu \in \operatorname{Ff} \mathfrak{s p O}(\mathcal{X})$ is fine fuzzy sp-Interior.

10. $F f_{\mathfrak{s p} C l}(v)=\wedge\left\{v: v \geq \mu, v \in \mathrm{Ff}_{\mathfrak{s p C}}(\mathcal{X})\right\}$ is fine fuzzy $\mathfrak{s p}$-closure.

Remark 3.1 A fine fuzzy set $\lambda$ of $\mathcal{X}$. Then

(i) $\quad \operatorname{FfsCl}(\lambda) \geq \lambda$ and $\operatorname{FfsInt}(\lambda) \leq \lambda$,

(ii) $\quad \lambda \leq \mu \Rightarrow \operatorname{FfsCl}(\lambda) \leq \operatorname{FfsCl}(\mu)$

(iii) $\quad \operatorname{FfInt}(\operatorname{FfCl}(\lambda)) \leq$ $F f \operatorname{sint}(\lambda) \leq F f \operatorname{sint}(\mu)$.

$\operatorname{FfsCl}(\lambda), \operatorname{Ffcl}(\operatorname{FfInt}(\lambda)) \geq \lambda$

\section{Proposition 3.1}

A fine fuzzy set $\lambda$ of $\mathcal{X}$, then following properties are true:

(i) $\operatorname{FfsCl}(\lambda) \geq \lambda \vee \operatorname{FfInt}(\operatorname{FfCl}(\lambda))$ and $\operatorname{FfsInt}(\lambda) \leq \lambda \wedge \operatorname{Ffcl}(\operatorname{FfInt}(\lambda))$

(ii) $\operatorname{FfpCl}(\lambda) \geq \lambda \vee \operatorname{Ffcl}(\operatorname{FfInt}(\lambda))$ and $\operatorname{Ffp} \operatorname{Int}(\lambda) \leq \lambda \wedge \operatorname{FfInt}(\operatorname{FfCl}(\lambda))$.

Proof

(i) Since, by the above remark it is easy.

(ii) Since, $\lambda \leq \operatorname{FfpCl}(\lambda), \operatorname{FfpCl}(\lambda)$ is a fine fuzzy pre closed set, $\quad \operatorname{Ffcl}(\operatorname{FfInt}(\lambda)) \leq$ $\operatorname{FfCl}(\operatorname{FfInt}(\mathrm{FfpCl}(\lambda))) \leq \mathrm{FfpCl}(\lambda)$.Thus, $\lambda$ V $\operatorname{FfCl}(\operatorname{FfInt}(\lambda)) \leq \operatorname{FfpCl}(\lambda)$.Since, $\quad \lambda \geq$ $\operatorname{FfpInt}(\lambda), \operatorname{FfpInt}(\lambda)$ is a fine fuzzy preopen set, $\operatorname{FfInt}(\operatorname{FfCl}(\lambda)) \geq$ FfInt $(\operatorname{FfCl}(\operatorname{Ffp} \operatorname{Int}(\lambda))) \geq \operatorname{FfpInt}(\lambda)$ Thus, $\lambda \wedge \operatorname{FfInt}(\operatorname{FfCl}(\lambda)) \geq \operatorname{FfpInt}(\lambda)$.

\section{Proposition 3.2}

For any fine fuzzy subset $\lambda$ of $\mathcal{X}$, then

(i) $\lambda$ is fine fuzzy sp-closed is equivalent to

Proof (i) $\Rightarrow$ (ii)

(ii) $\lambda \geq \operatorname{FfpCl}(\lambda) \wedge \operatorname{FfsCl}(\lambda)$

Given that $\lambda$ is a fine fuzzy $\mathfrak{s p}$-closed,

(i.e) $\lambda \geq \operatorname{FfInt}(\operatorname{FfCl}(\lambda)) \wedge \operatorname{FfCl}(\operatorname{FfInt}(\lambda))$.

Then $\operatorname{FfpCl}(\lambda) \wedge \operatorname{FfsCl}(\lambda) \geq \lambda \vee \operatorname{FfCl}(\operatorname{FfInt}(\lambda)) \wedge$ $\lambda \vee \operatorname{FfInt}(\operatorname{FfCl}(\lambda))$.

$\geq \lambda \vee$

$[\operatorname{FfCl}(\operatorname{FfInt}(\lambda)) \wedge \operatorname{FfInt}(\operatorname{FfCl}(\lambda))]$.

$$
\geq \lambda \vee \lambda=\lambda \text {. }
$$

Hence (i) $\Rightarrow$ (ii)

Conversely, (ii) $\Rightarrow(\mathrm{i})$. Assume that $\lambda \leq \operatorname{FfpInt}(\lambda) \vee$ $\operatorname{Ffs} \operatorname{Int}(\lambda)$. Since, by Definition 3.6 we get $\lambda$ is fine fuzzy $\mathfrak{s p -}$ closed.

Published By:

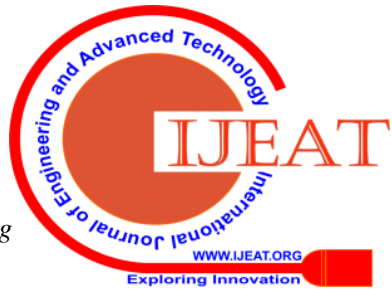




\section{Proposition 3.3}

For any two fine fuzzy sets $\lambda$ and $\mu$ of $\mathcal{X}$. Hence the following are true.

(i) $\operatorname{Ff}_{\mathfrak{s p C l}}(\lambda)$ is fine fuzzy $\mathfrak{s p}$ closed.

(ii) $\lambda \subseteq \operatorname{Ff} \mathfrak{s p C}(\mathcal{X}) \Leftrightarrow \lambda=\operatorname{Ff} \mathfrak{s p C l}(\lambda)$.

(iii) $\lambda \leq \mu \Rightarrow \operatorname{Ff}_{\mathfrak{s p C l}}(\lambda) \leq \operatorname{Ff} \mathfrak{s p C l}(\mu)$.

(iv) $\operatorname{Ff} \mathfrak{s p} \operatorname{Int}(\lambda)$ is fine fuzzy $\mathfrak{s p}$ open.

(v) $\lambda \subseteq \operatorname{Ff} \mathfrak{s p O}(\mathcal{X}) \Leftrightarrow \lambda=\operatorname{FfspInt}(\lambda)$.

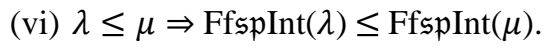

(vii) $\operatorname{FfInt}(\lambda) \leq \operatorname{Ff} \mathfrak{s p I n t}(\lambda) \leq \lambda \leq \operatorname{Ff} \mathfrak{s p C l}(\lambda) \leq \operatorname{FfCl}(\lambda)$.

\section{Proof}

(i) Assume that $\mu$ is $\operatorname{Ff} \mathfrak{s p C l}(\lambda)$ by the Definition 3.4, $\mu=\operatorname{FfspCl}(\lambda)=\wedge\{\eta: \eta \geq \lambda, \eta \in \operatorname{Ff} \mathfrak{s p C}(\mathcal{X})\}$.

Hence, $\mu$ is fine fuzzy $\mathfrak{s p}$ closed.

(ii) Assume that $\lambda=\operatorname{FfspCl}(\lambda)$ then by the Definition 3.4 we have,

$\lambda=\operatorname{Ff}_{\mathfrak{s p C l}}(\lambda)=\Lambda\{\mu: \mu \geq \lambda, \mu \in \operatorname{Ff} \mathfrak{s p C}(\mathcal{X})\}$

$\Leftrightarrow \lambda \in \wedge\{\mu: \mu \geq \lambda, \mu \in \operatorname{Ff} \mathfrak{s p C}(X)\}$.

$\Leftrightarrow \lambda$ is $\operatorname{Ff} \mathfrak{s p C}(X)$.

(iii) Assume that $\lambda \leq \mu$ then $\operatorname{FfspCl}(\lambda) \leq \operatorname{Ff} \mathfrak{s p C l}(\mu)$.

(iv) Assume that $\lambda$ is $F f_{s p} \operatorname{Int}(\lambda)$ by Definition 3.3 we get

$\operatorname{Ff} \mathfrak{s p} \operatorname{Int}(\lambda)=\vee\left\{\mu: \mu \leq \lambda, \mu \in \operatorname{Ff}_{\mathfrak{s p}} O(X)\right\}$. Hence $\lambda$ is fine fuzzy sp - open.

(v) Assume that $\lambda=F_{\mathfrak{s p}} \operatorname{Int}(\lambda)$ then by Definition 3.3 we have,

$\operatorname{Ff} \mathfrak{s p I n t}(\lambda)=\vee\{\mu: \mu \leq \lambda, \mu \in \operatorname{Ff} \mathfrak{s p} O(\mathcal{X})\}$.

$\Leftrightarrow \lambda \in \mathrm{V}\{\mu: \mu \leq \lambda, \mu \in \operatorname{FfspO}(\mathcal{X})\}$

$\Leftrightarrow \lambda \subseteq \operatorname{FfspO}(\mathcal{X})$.

(vi) Assume that $\lambda \leq \mu$ then $\operatorname{Ff} \mathfrak{s p I n t}(\lambda) \leq \operatorname{Ff} \mathfrak{F p} \operatorname{Int}(\mu)$.

(vii) Proof is immediate from Definition 3.6

\section{Lemma 3.1}

A fine fuzzy set $\lambda \in \mathcal{F} f T S(\mathcal{X})$. Then

(i) $\operatorname{FfCl}\left(1_{\chi}-\lambda\right)=1_{\chi}-\operatorname{FfInt}(\lambda)$ and

(ii) $\operatorname{FfInt}\left(1_{x}-\lambda\right)=1_{x}-\operatorname{FfCl}(\lambda)$.

\section{Proof}

(i) Let $\lambda$ be fine fuzzy set and $\mu$ be fine fuzzy open set with $\mu \leq \lambda$. Let $v \geq 11^{-\lambda}$ be fine fuzzy closed set. Then $\operatorname{FfInt}(\lambda)=v\left\{1_{\chi}-v: v \in \operatorname{Ff}_{\mathfrak{s p C}}(X)\right.$ and $\left.v \geq 1_{\chi}-\lambda\right\}$

$=1_{x}-\Lambda\left\{v: v \in\right.$ fine fuzzy closed set and $\left.v \geq 1_{x}-\lambda\right\}$ $\operatorname{FfInt}(\lambda)=1_{x}-\operatorname{FfCl}\left(1_{x}-\lambda\right)$.

Thus, $\operatorname{FfCl}\left(1_{x}-\lambda\right)=1_{x}-\operatorname{FfInt}(\lambda)$.

(ii) Let $\mu$ be a fine fuzzy set and $\lambda$ be fine fuzzy closed with $\lambda \leq \mu$. Hence, for a fine fuzzy open set $v \leq 1_{x}-$ $\lambda$.

$\operatorname{FfCl}(\lambda)=\wedge\left\{1_{\chi}-\mu: v \in \operatorname{Ff}_{\mathfrak{s p}} O(X) v \leq 1_{\chi}-\lambda\right\}$

$=1_{x}-\mathrm{v}\left\{\mu\right.$ : $\mu$ is a fine fuzzy open set and $\left.v \leq 1_{x}-\lambda\right\}$ $\operatorname{FfCl}(\lambda)=1_{x}-\operatorname{FfInt}\left(1_{x}-\lambda\right)$.Thus, $\operatorname{FfCl}\left(1_{x}-\lambda\right)=1_{x}-$ $\operatorname{FfInt}(\lambda)$

\section{Lemma 3.2}

A fine fuzzy set $\lambda \in \mathcal{F} f \operatorname{TS}(\mathcal{X})$,

(i) $\operatorname{FfspCl}\left(1_{x}-\lambda\right)=1_{x}-\operatorname{Ffsp} \operatorname{Int}(\lambda)$ and

(ii) $\operatorname{Ffsp} \operatorname{Int}\left(1_{x}-\lambda\right)=1_{x}-\operatorname{Ff} \mathfrak{s p C l}(\lambda)$. (i) . A fine fuzzy set $\lambda, \mu$ is fine fuzzy $\mathfrak{s p}$ open set with $\mu \leq \lambda$. Let $v \geq 1_{x}-\lambda$ be fine fuzzy $\mathfrak{s p}$ closed set. Then

$\operatorname{Ff} \mathfrak{s p}_{\operatorname{Int}}(\lambda)=v\left\{1_{\chi}-v: v \in \operatorname{Ff} \mathfrak{s p C}(\mathcal{X})\right.$ and $\left.v \geq 1_{\chi}-\lambda\right\}$

$=1_{X}-\Lambda\left\{v: v \in \operatorname{FfspC}(\mathcal{X})\right.$ and $\left.v \geq 1_{x}-\lambda\right\}$.

$\operatorname{Ffsp} \operatorname{Int}(\lambda)=1_{x}-\operatorname{FfspCl}\left(1_{x}-\lambda\right)$.

Thus, $\operatorname{Ff} \mathfrak{s p C l}\left(1_{x}-\lambda\right)=1_{x}-\operatorname{Ffsp} \operatorname{Int}(\lambda)$.

(ii). A fine fuzzy set $\mu, \lambda \in$ fine fuzzy $\mathfrak{s p}$ closed with $\lambda \leq \mu$. Then for a fine fuzzy $\mathfrak{s p}$ open set $v \leq 1_{\chi}-$ $\lambda$.

$\begin{aligned} \operatorname{FfspCl}(\lambda) & =\wedge\left\{1_{\chi}-\mu: v \in \operatorname{Ff}_{\mathfrak{s p O}}(X), v \leq 1_{\chi}-\lambda\right\} \\ & =1_{X}-v\left\{v \in \operatorname{Ff}_{\mathfrak{s p} O}(X), v \leq 1_{\chi}-\lambda\right\}\end{aligned}$

$\operatorname{Ff} \mathfrak{s p C l}(\lambda)=1_{x}-\operatorname{Ff} \mathfrak{s p I n t}\left(1_{x}-\lambda\right)$.

Thus, $\operatorname{Ff} \mathfrak{s p I n t}\left(1_{x}-\lambda\right)=1_{x}-\operatorname{Ff} \mathfrak{s p C l}(\lambda)$.

\section{Proposition 3.4}

A fine fuzzy set $\lambda$ of $\mathcal{X}$. Thus the following statements are hold

$(\mathrm{i}) \operatorname{FfspCl}(\lambda) \geq \lambda \vee((\operatorname{FfInt}(\operatorname{FfCl}(\lambda)) \wedge(\operatorname{FfCl}(\operatorname{FfInt}(\lambda))))$.

(ii) $\operatorname{FfspInt}(\lambda) \leq \lambda \wedge((\operatorname{FfInt}(\operatorname{FfCl}(\lambda)) \vee(\operatorname{FfCl}(\operatorname{FfInt}(\lambda))))$

(iii) $\operatorname{FfspCl}(\lambda) \geq(\operatorname{FfsCl}(\lambda)) \wedge(\operatorname{Ffpcl}(\lambda))$.

(iv) $\operatorname{Ff} \mathfrak{s p} \operatorname{Int}(\lambda) \leq(\operatorname{Ffs} \operatorname{Int}(\lambda)) \vee(\operatorname{FfpInt}(\lambda))$

\section{Proof}

(i)

Since, $\operatorname{Ff} \mathfrak{s p c l}(\lambda)$ is fine fuzzy $\mathfrak{s p}$ closed set (i.e)

$\lambda \geq \operatorname{FfInt}(\operatorname{FfCl}(\lambda)) \wedge(\operatorname{FfCl}(\operatorname{FfInt}(\lambda)))$ and $\lambda \leq \operatorname{Ff} \mathfrak{s p C l}(\lambda)$.

$\operatorname{Ff} \mathfrak{s p C l}(\lambda) \geq \lambda \geq \operatorname{FfInt}(\operatorname{FfCl}(\lambda)) \wedge(\operatorname{FfCl}(\operatorname{FfInt}(\lambda)))$.

Hence, $\operatorname{Ff} \mathfrak{s p C l}(\lambda) \geq \lambda \vee((\operatorname{FfInt}(\operatorname{FfCl}(\lambda)) \wedge$

$(\operatorname{FfCl}(\operatorname{FfInt}(\lambda))))$

(ii) Since, $F f_{\mathfrak{s p}} \operatorname{Int}(\lambda)$ is fine fuzzy $\mathfrak{s p}$ open set (i.e) $\lambda \leq \operatorname{FfInt}(\operatorname{FfCl}(\lambda)) \vee(\operatorname{FfCl}(\operatorname{FfInt}(\lambda)))$ and $\lambda \leq \operatorname{FfspInt}(\lambda)$. $\operatorname{Ff} \mathfrak{s p I n t}(\lambda) \leq \lambda \leq \operatorname{FfInt}(\operatorname{FfCl}(\lambda)) \vee(\operatorname{FfCl}(\operatorname{FfInt}(\lambda)))$.

Hence, $\operatorname{Ff} \mathfrak{s p}_{\operatorname{Int}}(\lambda) \leq \lambda \wedge$

$((\operatorname{FfInt}(\operatorname{FfCl}(\lambda))(\operatorname{FfCl}(\operatorname{FfInt}(\lambda))))$.

(iii)Assume

$$
\begin{gathered}
(\operatorname{FfsCl}(\lambda)) \wedge(\operatorname{FfpCl}(\lambda)) \geq(\lambda \vee \operatorname{FfInt}(\operatorname{FfsCl}(\lambda))) \wedge \\
(\lambda \vee(\operatorname{FfCl}(\operatorname{FfInt}(\lambda)))) \\
=\lambda \vee((\operatorname{FfInt}(\operatorname{FfCl}(\lambda)) \wedge(\operatorname{FfCl}(\operatorname{FfInt}(\lambda)))) \\
=(\operatorname{FfInt}(\operatorname{FfCl}(\lambda)) \wedge(\operatorname{FfCl}(\operatorname{FfInt}(\lambda)))) \geq
\end{gathered}
$$

Thus, $(\operatorname{FfsCl}(\lambda)) \wedge(\operatorname{FfpCl}(\lambda)) \geq \operatorname{FfspCl}(\lambda)$.

(iv) $(\operatorname{Ffs} \operatorname{Int}(\lambda)) \vee(\operatorname{FfpInt}(\lambda)) \leq(\lambda \wedge \operatorname{FfCl}(\operatorname{FfInt}(\lambda))) \vee$

$(\lambda \wedge \operatorname{FfInt}(\operatorname{FfCl}(\lambda)))=\lambda \wedge$

$(((\operatorname{FfCl}(\operatorname{FfInt}(\lambda))) \vee \operatorname{FfInt}(\operatorname{FfCl}(\lambda)))$

\section{Proof}




$$
=((\operatorname{FfCl}(\operatorname{FfInt}(\lambda))) \vee \operatorname{FfInt}(\operatorname{FfCl}(\lambda))) \leq \operatorname{Ff} \mathfrak{s p i n t}(\lambda) .
$$

\section{Proposition 3.5}

Let $\lambda$ be a fine fuzzy subset of $\mathcal{X}$. Then the equivalent statements are valid

(i) $\lambda \in$ fine fuzzy $\mathfrak{s p}$ closed.

(ii) $\lambda^{c} \in$ fine fuzzy $\mathfrak{s p}$ open.

(iii) $\lambda \geq((F f \operatorname{Int}(F f C l(\lambda)) \wedge(F f C l(F f \operatorname{Int}(\lambda))))$.

(iv) $\lambda^{c} \leq((F f \operatorname{Int}(F f C l(\lambda)) \vee(F f C l(F f \operatorname{Int}(\lambda))))$.

Proof (i) $\Leftrightarrow$ (ii) follows from Lemma 3.2

(i) $\Rightarrow$ (iii)

By definition $\exists$ a fine fuzzy sp closed set

$\lambda \geq((F f \operatorname{Int}(F f C l(\lambda)) \wedge(F f C l(F f \operatorname{Int}(\lambda))))$.

(i) $\Rightarrow$ (iv)

By definition $\exists$ a fine fuzzy $\mathfrak{s p}$ closed set :

$\lambda \geq((F f \operatorname{Int}(F f C l(\lambda)) \wedge(F f C l(F f \operatorname{Int}(\lambda))))$ and $1_{X}-\lambda$ is fine fuzzy $\mathfrak{s p}$ open set. Hence, $\lambda^{c} \leq((\operatorname{FfInt}(\operatorname{FfCl}(\lambda)) \vee(\operatorname{FfCl}(\operatorname{FfInt}(\lambda))))$.

\section{Proposition 3.6}

A fine fuzzy set $\lambda \in \mathcal{F} f \operatorname{TS}(\mathcal{X})$. Hence, the properties are hold

(i) $\quad \operatorname{Ff} \mathfrak{s p C l}\left(0_{X}\right)=0_{X}$.

(ii) $\quad \operatorname{Ff} \mathfrak{s p C l}(\operatorname{Ff} \mathfrak{s p C l}(\lambda))=\operatorname{Ff} \mathfrak{s p C l}(\lambda)$.

(iii) $\quad \operatorname{Ff} \mathfrak{s p} \operatorname{Int}(\operatorname{Ff} \mathfrak{s p} \operatorname{Int}(\lambda))=\operatorname{Ff} \mathfrak{s p} \operatorname{Int}(\lambda)$.

\section{Proof}

It is obvious.

\section{Proposition 3.7}

A $\mathcal{F} f T S(\mathcal{X})$, the relations are valid

(i) $\quad \operatorname{Ff} \mathfrak{s p C l}(\lambda \vee \mu) \geq \operatorname{Ff} \mathfrak{s p C l}(\lambda) \vee \operatorname{Ff}_{\mathfrak{s p C l}}(\mu)$

(ii) $\quad \operatorname{Ff}_{\mathfrak{s p C l}}(\lambda \wedge \mu) \leq \operatorname{Ff}_{\mathfrak{s p}} \mathrm{Cl}(\lambda) \wedge \operatorname{Ff} \mathfrak{s p C l}(\mu)$.

(iii) $\quad \operatorname{Ff} \mathfrak{s p I n t}(\lambda \vee \mu) \geq \operatorname{Ff}_{\mathfrak{s p} \operatorname{Int}(\lambda) \vee} \mathrm{Ff}_{\mathfrak{s p} \operatorname{Int}}(\mu)$.

(iv) $\quad \operatorname{Ff}$ spInt $(\lambda \wedge \mu) \leq \operatorname{Ffsp}_{\operatorname{Int}}(\lambda) \wedge \operatorname{Ff} \mathfrak{s p I n t}(\mu)$

\section{Proof}

(i) $\lambda \leq \lambda \vee \mu$ or $\mu \leq \lambda \vee \mu$ that implies $\operatorname{Ff} \mathfrak{s p C l}(\lambda) \leq \operatorname{Ff} \mathfrak{s p C l}(\lambda \vee \mu)$ or

$\operatorname{Ff} \mathfrak{s p C l}(\mu) \leq \operatorname{Ff} \mathfrak{s p C l}(\lambda \vee \mu)$. Therefore,

$\operatorname{Ff} \mathfrak{s p C l}(\lambda \vee \mu) \geq \operatorname{Ff} \mathfrak{s p C l}(\lambda) \vee \operatorname{Ff} \mathfrak{s p C l}(\mu)$.

$\operatorname{Ff} \mathfrak{s p C l}(\lambda) \geq \operatorname{Ff} \mathfrak{s p C l}(\lambda \wedge \mu)$ or

$\operatorname{Ff} \mathfrak{s p C l}(\mu) \geq \operatorname{Ff} \mathfrak{s p C l}(\lambda \wedge \mu)$.

Therefore, $\operatorname{Ff} \mathfrak{s p C l}(\lambda \wedge \mu) \geq \operatorname{Ff} \mathfrak{s p C l}(\lambda) \wedge \operatorname{Ff} \mathfrak{s p C l}(\mu)$. (iii) $\lambda \leq \lambda \vee \mu$ or $\mu \leq \lambda \vee \mu$ that implies $\operatorname{Ff} \mathfrak{s p I n t}(\lambda) \leq \mathrm{Ff} \mathfrak{s p} \operatorname{Int}(\lambda \vee \mu)$ or

FfspInt $(\mu) \leq \mathrm{Ff} \mathfrak{s p I n t}(\lambda \vee \mu)$. Therefore,

$\operatorname{Ff} \mathfrak{s p I n t}(\lambda \vee \mu) \geq \operatorname{Ff}_{\mathfrak{s p} \operatorname{Int}}(\lambda) \vee \operatorname{Ff} \mathfrak{s p} \operatorname{Int}(\mu)$. $\operatorname{Ff} \mathfrak{s p I n t}(\lambda) \geq \operatorname{Ff} \mathfrak{s p I n t}(\lambda \wedge \mu)$ or

$\operatorname{Ff} \mathfrak{s p I n t}(\mu) \geq \mathrm{Ff}_{\mathfrak{s p} I n t}(\lambda \wedge \mu)$. Therefore, $\operatorname{Ff} \mathfrak{s p I n t}(\lambda \wedge \mu) \geq \operatorname{Ff} \mathfrak{s p I n t}(\lambda) \wedge \operatorname{Ff} \mathfrak{s p I n t}(\mu)$. (ii) $\quad \lambda \geq \lambda \wedge \mu$ or $\mu \geq \lambda \wedge \mu$ that implies

(iv) $\lambda \geq \lambda \wedge \mu$ or $\mu \geq \lambda \wedge \mu \quad$ that implies

\section{RESULT DESCRIPTIONS}

\section{Proposition 4.1}

$$
\text { Every } F f C \text { is } F f s C \text {. }
$$

\section{Proof}

For a fine fuzzy closed set $\lambda, \operatorname{FfCl}(\lambda)=\lambda$.

To prove $\lambda$ is fine fuzzy semi closed

Since, $\operatorname{FfCl}(\lambda) \leq \lambda$ and $\operatorname{FfInt}(\lambda) \leq \lambda$

$F f \operatorname{Int}((F f C l(\lambda))) \leq F f \operatorname{Int}(\lambda)$

$F f \operatorname{Int}(f F C l(\lambda)) \leq \lambda$.

Hence, every $F f C$ set is $F f s C$.

\section{Note 4.1}

The converse of Proposition 4.1 need not be true, shows in the following

\section{Example 4.1}

Let $\mathcal{X}=\{\mathrm{a}, \mathrm{b}\}$ be a nonempty set. Let $\lambda_{1}, \lambda_{2}, \lambda_{3}$, $\lambda_{4}, \lambda_{5}, \lambda_{6} \in I^{X}$ be defined as $\lambda_{1}(a)=0.3, \lambda_{1}(b)=0.5$; $\lambda_{2}(a)=0.6, \quad \lambda_{2}(b)=0.5 ; \quad \lambda_{3}(a)=0.4, \quad \lambda_{3}(b)=0.4$; $\lambda_{4}(a)=0.3, \quad \lambda_{4}(b)=0.4 ; \quad \lambda_{5}(a)=0.5, \quad \lambda_{5}(b)=0.6$; $\lambda_{6}(a)=0.5, \lambda_{6}(b)=0.5$. Let $\mathcal{T}=\left\{0,1, \lambda_{1}, \lambda_{2}\right\}$ be the fuzzy topology on $\mathcal{X}$ and Then $\mathcal{T}_{f}=\left\{0_{\mathrm{X}}, 1_{\mathrm{X}}, \bigcup_{\alpha \in \mathrm{J}}\left\{\mathrm{T}_{\alpha}\right\}\right\}$ fine fuzzy topology defined as $\mathcal{T}\left(\lambda_{\alpha}\right)=\mathcal{T}_{\alpha} \mathcal{T}\left(\lambda_{1}\right)=\mathcal{T}_{1}=$ $\lambda_{3}=\left\{\lambda_{3} \wedge \lambda_{1}=\lambda_{4} \neq 0\right\}, \mathcal{T}\left(\lambda_{2}\right)=\mathcal{T}_{1}=\lambda_{5}=$ $\left\{\lambda_{5} \wedge \lambda_{2}=\lambda_{6} \neq 0\right\}$. Thus, $\left(\mathcal{X}, \mathcal{T}, \mathcal{T}_{f}\right)$ is $\mathcal{F} f T S$. Let $\lambda_{3} \in$ FfsC, but $\lambda_{3} \notin F f C$.

\section{Proposition 4.2}

Every $F f C$ is $F f \alpha C$.

\section{Proof}

Let $\lambda$ be fine fuzzy closed, $\operatorname{FfCl}(\lambda)=\lambda$.

To prove: $\lambda \in F f \alpha C$.

Since, $\lambda \leq \operatorname{FfCl}(\lambda)$

$\operatorname{FfInt}(\lambda) \leq(\operatorname{FfInt}(\operatorname{FfCl}(\lambda)))$

$\operatorname{FfCl}(F f \operatorname{Int}(\lambda)) \leq F f C l(F f \operatorname{Int}(F f C l(\lambda)))$

$\operatorname{FfInt}(\lambda) \leq F f C l(F f \operatorname{Int}(F f C l(\lambda)))$

$\lambda \leq F f C l(F f \operatorname{Int}(F f C l(\lambda)))$.

Hence, every $F f C$ is $F f \alpha C$.

\section{Note 4.2}

The converse of Proposition 4.2 need not be true shows in the following

\section{Example 4.2}

Let $X=\{\mathrm{a}, \mathrm{b}\}$ be a nonempty set. Let $\lambda_{1}, \lambda_{2}, \lambda_{3}$, $\lambda_{4}, \lambda_{5}, \lambda_{6} \in I^{X}$ be defined as $\lambda_{1}(a)=0.3, \lambda_{1}(b)=0.5$; $\lambda_{2}(a)=0.6, \quad \lambda_{2}(b)=0.5 ; \quad \lambda_{3}(a)=0.4, \quad \lambda_{3}(b)=0.4$; $\lambda_{4}(a)=0.3, \quad \lambda_{4}(b)=0.4 ; \quad \lambda_{5}(a)=0.5, \quad \lambda_{5}(b)=0.6 ;$ $\lambda_{6}(a)=0.5, \lambda_{6}(b)=0.5$. Then $(\mathcal{X}, \mathcal{T})$ is fTS where $\mathcal{T}=$ $\left\{0,1, \lambda_{1}, \lambda_{2}\right\}$ and $\mathcal{T}_{f}=\left\{0_{X}, 1_{X}, \bigcup_{\alpha \in J}\left\{T_{\alpha}\right\}\right\}$ a fine fuzzy topology defined as $\mathcal{T}\left(\lambda_{1}\right)=\mathcal{T}_{1}=\lambda_{3}=$ $\left\{\lambda_{3} \wedge \lambda_{1}=\lambda_{4} \neq\right.$ 
$0\}, \quad \mathcal{T}\left(\lambda_{2}\right)=\mathcal{T}_{2}=\lambda_{5}=\left\{\lambda_{5} \wedge \lambda_{2}=\lambda_{6} \neq 0\right\}$. Thus, $(x$, $\left.\mathcal{T}, \mathcal{T}_{f}\right)$ is $\mathcal{F} f T S$. Let $\lambda_{3} \in F f \alpha C$, but $\lambda_{3} \notin F f C$.

\section{Proposition 4.3}

Every $F f S C$ is $F f \mathfrak{s p} C$.

\section{Proof}

Let $\lambda \in F f s C$.

Since, $F f \operatorname{Int}(F f C l(\lambda)) \leq \lambda$

Since, $F f \operatorname{Int}(\lambda) \leq \lambda$

$\operatorname{FfCl}(F f \operatorname{Int}(\lambda)) \leq F f c l(\lambda)$

$\operatorname{FfCl}(F f \operatorname{Int}(\lambda)) \leq \lambda$

From (1) and (2)

$\lambda \wedge F f C l(\lambda) \geq F f \operatorname{Int}(F f C l(\lambda)) \wedge F f C l(F f \operatorname{Int}(\lambda))$

$\lambda \geq F f \operatorname{Int}(F f C l(\lambda)) \wedge F f C l(F f \operatorname{Int}(\lambda))$.

Hence, every $F f_{s} C$ is $F f_{\mathfrak{s p} C}$.

\section{Note 4.3}

The converse of Proposition 4.3 need not be true shows in the following

\section{Example 4.3}

Let $\mathcal{X}=\{\mathrm{a}, \mathrm{b}\}$ be a nonempty set. Let $\lambda_{1}, \lambda_{2}, \lambda_{3}$, $\lambda_{4}, \lambda_{5}, \lambda_{6} \in I^{X}$ be defined as $\lambda_{1}(a)=0.3, \lambda_{1}(b)=0.5$; $\lambda_{2}(a)=0.6, \quad \lambda_{2}(b)=0.5 ; \quad \lambda_{3}(a)=0.4, \quad \lambda_{3}(b)=0.4$; $\lambda_{4}(a)=0.3, \quad \lambda_{4}(b)=0.4 ; \quad \lambda_{5}(a)=0.5, \quad \lambda_{5}(b)=0.6$; $\lambda_{6}(a)=0.5, \lambda_{6}(b)=0.5$. Let $\mathcal{T}=\left\{0,1, \lambda_{1}, \lambda_{2}\right\}$ be the fuzzy topology on $X$ and Then $\mathcal{T}_{f}=\left\{0_{X}, 1_{X}, \bigcup_{\alpha \in J}\left\{T_{\alpha}\right\}\right\}$

be fine fuzzy topology defined as $\mathcal{T}\left(\lambda_{1}\right)=\mathcal{T}_{1}=\lambda_{3}=$ $\left\{\lambda_{3} \wedge \lambda_{1}=\lambda_{4} \neq 0\right\}, \mathcal{T}\left(\lambda_{2}\right)=\mathcal{T}_{2}=\lambda_{5}=\left\{\lambda_{5} \wedge \lambda_{2}=\right.$ $\left.\lambda_{6} \neq 0\right\}$. Thus, $\left(\mathcal{X}, \mathcal{T}, \mathcal{T}_{f}\right)$ is $\mathcal{F} f T S$. Let $\lambda_{6} \in F f_{\mathfrak{s p} C}$ but $\lambda_{6} \notin F f s C$.

\section{Proposition 4.4}

Every $F f p C$ is $F f \mathfrak{s p} C$.

\section{Proof}

Let $\lambda$ be fine fuzzy pre-closed set.

Since, $\lambda \geq F f C l(F f \operatorname{Int}(\lambda))$ and $\lambda \geq F f \operatorname{Int}(F f C l(\lambda))$.

$\Rightarrow \lambda \geq F f C l(F f \operatorname{Int}(\lambda)) \wedge F f \operatorname{Int}(F f C l(\lambda))$

Hence, every $F f p C$ is $F f \mathfrak{s p} C$.

\section{Note 4.4}

The converse of Proposition 4.4 need not be true shows in the following

\section{Example 4.4}

Let $X=\{\mathrm{a}, \mathrm{b}\}$ be a nonempty set. Let $\lambda_{1}, \lambda_{2}, \lambda_{3}$, $\lambda_{4}, \lambda_{5}, \lambda_{6} \in I^{x}$ be defined as $\lambda_{1}(a)=0.3, \lambda_{1}(b)=0.5$; $\lambda_{2}(a)=0.6, \quad \lambda_{2}(b)=0.5 ; \quad \lambda_{3}(a)=0.4, \quad \lambda_{3}(b)=0.4$; $\lambda_{4}(a)=0.3, \quad \lambda_{4}(b)=0.4 ; \quad \lambda_{5}(a)=0.5, \quad \lambda_{5}(b)=0.6$; $\lambda_{6}(a)=0.5, \lambda_{6}(b)=0.5$. Let $\mathcal{T}=\left\{0,1, \lambda_{1}, \lambda_{2}\right\}$ be the be fine fuzzy topology defined as $\mathcal{T}\left(\lambda_{1}\right)=\mathcal{T}_{1}=\lambda_{3}=$ $\left\{\lambda_{3} \wedge \lambda_{1}=\lambda_{4} \neq 0\right\}, \mathcal{T}\left(\lambda_{2}\right)=\mathcal{T}_{2}=\lambda_{5}=\left\{\lambda_{5} \wedge \lambda_{2}=\right.$ $\left.\lambda_{6} \neq 0\right\}$. Thus, $\left(\mathcal{X}, \mathcal{T}, \mathcal{T}_{f}\right)$ is $\mathcal{F} f T S$. Let $\lambda_{5} \in F f_{\mathfrak{s p} C}$, but $\lambda_{5} \notin F f p C$. fuzzy topology on $\mathcal{X}$ and Then $\mathcal{T}_{f}=\left\{0_{X}, 1_{X}, \bigcup_{\alpha \in J}\left\{\mathrm{~T}_{\alpha}\right\}\right\}$

\section{Every $\mathrm{Ff} \mathfrak{s p C}$ is $F f \beta C$.}

Proof

Let $\lambda$ be fine fuzzy $\mathfrak{s p - c l o s e d ~ s e t . ~ i . e ~} \lambda \geq$ $\operatorname{FfCl}(F f \operatorname{Int}(\lambda)) \wedge F f \operatorname{Int}(F f C l(\lambda))$.

To Prove $\lambda \geq F f \operatorname{Int}(F f C l(F f \operatorname{Int}(\lambda)))$.

Also, $\lambda \geq F f C l(F f \operatorname{Int}(\lambda))$ and $\lambda \geq F f \operatorname{Int}(F f C l(\lambda))$.

$\lambda \geq F f \operatorname{Int}(\lambda) \geq F f \operatorname{Int}(F f C l(F f \operatorname{Int}(\lambda)))$.

Hence, every FfapC is $F f \beta C$.

\section{Note 4.5}

The converse of Proposition 4.5 need not be true shows in the following

\section{Example 4.5}

Let $\mathcal{X}=\{\mathrm{a}, \mathrm{b}\}$ be a nonempty set. Let $\lambda_{1}, \lambda_{2}, \lambda_{3}$, $\lambda_{4}, \lambda_{5}, \lambda_{6}, \lambda_{7} \in I^{X}$ be defined as $\lambda_{1}(a)=0.3, \lambda_{1}(b)=$ $0.5 ; \lambda_{2}(a)=0.6, \lambda_{2}(b)=0.5 ; \lambda_{3}(a)=0.4, \lambda_{3}(b)=0.4$; $\lambda_{4}(a)=0.3, \quad \lambda_{4}(b)=0.4 ; \quad \lambda_{5}(a)=0.5, \quad \lambda_{5}(b)=0.6$; $\lambda_{6}(a)=0.5, \lambda_{6}(b)=0.5 ; \lambda_{7}(a)=0.6, \lambda_{7}(b)=0.7$. Let $\mathcal{T}=\left\{0,1, \lambda_{1}, \lambda_{2}\right\}$ be the fuzzy topology on $\mathcal{X}$ and Then $\mathcal{T}_{f}$ $=\left\{0_{X}, 1_{X}, \bigcup_{\alpha \in J}\left\{T_{\alpha}\right\}\right\}$ be fine fuzzy topology defined as $\mathcal{T}\left(\lambda_{1}\right)=\mathcal{T}_{1}=\lambda_{3}=\left\{\lambda_{3} \wedge \lambda_{1}=\lambda_{4} \neq 0\right\}, \mathcal{T}\left(\lambda_{2}\right)=\mathcal{T}_{2}=$ $\lambda_{5}=\left\{\lambda_{5} \wedge \lambda_{2}=\lambda_{6} \neq 0\right\}$. Thus, $\left(\mathcal{X}, \mathcal{T}, \mathcal{T}_{f}\right)$ is $\mathcal{F} f T S$. Let $\lambda_{7}$ $\in F f \beta C$, but $\lambda_{7} \notin F f \mathfrak{s p} C$.

\section{Proposition 4.6}

Every $F f p C$ is $F f \beta C$.

Proof

Let $\lambda \in F f p C$.

Since, $F f C l(F f \operatorname{Int}(\lambda)) \leq \lambda$

To Prove $F f \operatorname{Int}(F f C l(F f \operatorname{Int}(\lambda))) \leq \lambda$.

$\Rightarrow F f \operatorname{Int}(F f C l(F f \operatorname{Int}(\lambda))) \leq F f \operatorname{Int}(\lambda) \leq \lambda$.

Hence, every $F f p C$ is $F f \beta C$.

\section{Note 4.6}

The converse of Proposition 4.6 need not be true shows in the following

\section{Example 4.6}

Let $\mathcal{X}=\{\mathrm{a}, \mathrm{b}\}$ be a nonempty set. Let $\lambda_{1}, \lambda_{2}, \lambda_{3}$, $\lambda_{4}, \lambda_{5}, \lambda_{6}, \lambda_{7} \in I^{x}$ be defined as $\lambda_{1}(a)=0.3, \lambda_{1}(b)=$ $0.5 ; \lambda_{2}(a)=0.6, \lambda_{2}(b)=0.5 ; \lambda_{3}(a)=0.4, \lambda_{3}(b)=0.4$ $\lambda_{4}(a)=0.3, \quad \lambda_{4}(b)=0.4 ; \quad \lambda_{5}(a)=0.5, \quad \lambda_{5}(b)=0.6$ $\lambda_{6}(a)=0.5, \lambda_{6}(b)=0.5 ; \lambda_{7}(a)=0.5, \quad \lambda_{7}(b)=1$. Let $T=\left\{0,1, \lambda_{1}, \lambda_{2}\right\}$ be the fuzzy topology on $\mathcal{X}$ and Then $\mathcal{T}_{f}$ $=\left\{0_{X}, 1_{X}, \bigcup_{\alpha \in J}\left\{T_{\alpha}\right\}\right.$ be fine fuzzy topology defined as $\mathcal{T}\left(\lambda_{1}\right)=\mathcal{T}_{1}=\lambda_{3}=\left\{\lambda_{3} \wedge \lambda_{1}=\lambda_{4} \neq 0\right\}, \mathcal{T}\left(\lambda_{2}\right)=\mathcal{T}_{2}=$ $\lambda_{5}=\left\{\lambda_{5} \wedge \lambda_{2}=\lambda_{6} \neq 0\right\}$. Thus, $\left(\mathcal{X}, \mathcal{T}, \mathcal{T}_{f}\right)$ is $\mathcal{F} f T S$. Let $\lambda_{7}$ $\in F f \beta C$, but $\lambda_{7} \notin F f p C$.

\section{Proposition 4.7}

Every $F f C$ is $F f \mathfrak{s p} C$.

Proof

Let $\lambda \in F f C(\mathcal{X})$ and by Proposition $3.9 \& 3.12$ we have

\section{Proposition 4.5}


Since, $F f \operatorname{Int}(\lambda) \leq \lambda$ and $F f C l(F f \operatorname{Int}(\lambda)) \leq(F f C l(\lambda))$

$$
F f C l(F f \operatorname{Int}(\lambda)) \leq(F f C l(\lambda)) \leq \lambda
$$

Since, $\lambda \geq F f C l(\lambda), F f \operatorname{Int}(\lambda) \geq F f \operatorname{Int}(F f C l(\lambda))$

$$
\lambda \geq F f \operatorname{Int}(F f C l(\lambda))
$$

Hence, from (1) and (2) $\lambda \in F f \mathfrak{s p} C$.

\section{Note 4.7}

The converse of Proposition 4.7 need not be true shows in the following

\section{Example 4.7}

Let $X=\{\mathrm{a}, \mathrm{b}\}$ be a nonempty set. Let $\lambda_{1}, \lambda_{2}, \lambda_{3}$, $\lambda_{4}, \lambda_{5}, \lambda_{6} \in I^{x}$ be defined as $\lambda_{1}(a)=0.3, \lambda_{1}(b)=0.5$; $\lambda_{2}(a)=0.6, \quad \lambda_{2}(b)=0.5 ; \quad \lambda_{3}(a)=0.4, \quad \lambda_{3}(b)=0.4$; $\lambda_{4}(a)=0.3, \quad \lambda_{4}(b)=0.4 ; \quad \lambda_{5}(a)=0.5, \quad \lambda_{5}(b)=0.6$; $\lambda_{6}(a)=0.5, \lambda_{6}(b)=0.5$. Let $\mathcal{T}=\left\{0,1, \lambda_{1}, \lambda_{2}\right\}$ be the fuzzy topology on $\mathcal{X}$ and Then $\mathcal{T}_{f}=\left\{0_{\mathrm{X}}, 1_{\mathrm{X}}, \bigcup_{\alpha \in \mathrm{J}}\left\{\mathrm{T}_{\alpha}\right\}\right\}$ be fine fuzzy topology defined as $\mathcal{T}\left(\lambda_{1}\right)=\mathcal{T}_{1}=\lambda_{3}=$ $\left\{\lambda_{3} \wedge \lambda_{1}=\lambda_{4} \neq 0\right\}, \mathcal{T}\left(\lambda_{2}\right)=\mathcal{T}_{2}=\lambda_{5}=\left\{\lambda_{5} \wedge \lambda_{2}=\right.$ $\left.\lambda_{6} \neq 0\right\}$. Thus, $\left(\mathcal{X}, \mathcal{T}, \mathcal{T}_{f}\right)$ is $\mathcal{F} f T S$. Let $\lambda_{5} \in F f_{\mathfrak{s p} C}$, but $\lambda_{5} \notin F f C$.

\section{Note 4.8}

Clearly the above discussions gives the following implications:

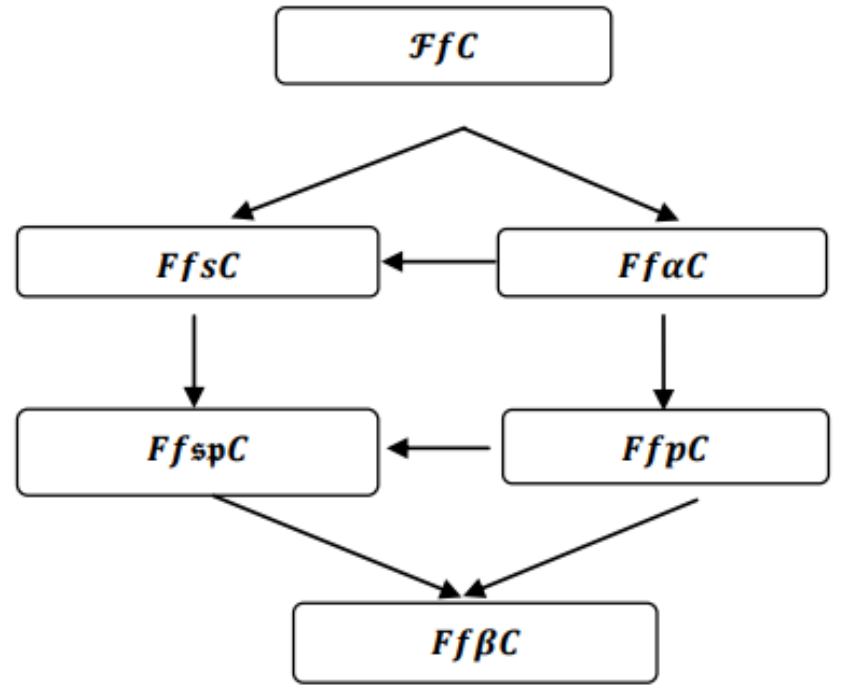

(figure - 4.1)

Interrelations between $\boldsymbol{F} f \mathfrak{s p} C$ and other types of fine fuzzy sets

\section{Proposition 4.8}

Let $\left(\mathcal{X}, \mathcal{T}, \mathcal{T}_{f}\right)$ be $\mathcal{F} f T S$. Then

(i) An arbitrary union of fine fuzzy $\mathfrak{s p}$ open sets are fine fuzzy open set.

(ii) A finite intersection of fine fuzzy $\mathfrak{s p}$-closed sets are not fine fuzzy closed.

\section{FINE FUZZY $\mathfrak{s p}$ CONTINUOUS FUNCTION}

\section{Definition 5.1}

A function $\psi:\left(\mathcal{X}, \mathcal{T}, \mathcal{T}_{f}\right) \rightarrow\left(\mathcal{Y}, \mathcal{S}, \mathcal{S}_{f}\right)$ is

a. $F f_{\mathfrak{s p} O}\left(F f_{\mathfrak{s p} C}\right)$ if $\psi^{-1}(v) \in F f_{\mathfrak{s p}} O(\mathcal{Y})$ $[F f \mathfrak{s p C}(\mathcal{Y})] \forall v \in F f O(X)[F f C(\mathcal{X})]$. b. $\quad F f \mathfrak{s p}^{*} O \quad\left(F f \mathfrak{s p}^{*} C\right)$ if $\psi(v) \in F f \mathfrak{s p} O(\mathcal{Y})$ $[F f \mathfrak{s p} C(\mathcal{Y})] \forall v \in F f O(X)[F f C(X)]$.

c. $\left.F f_{\mathfrak{p p}^{* *}} O\left(F f_{\mathfrak{p p}^{* *}}\right)^{*}\right)$ if $\psi(v) \quad \in F f O(X)$ $[F f C(\mathcal{X})] \forall v \in F f_{\mathfrak{s p} O}(\mathcal{Y})\left[F f_{\mathfrak{s p} C}(\mathcal{Y})\right]$.

\section{Definition 5.2}

Let $\quad\left(\mathcal{X}, \mathcal{T}, \mathcal{T}_{f}\right)$ and $\quad\left(\mathcal{Y}, \mathcal{S}, \mathcal{S}_{f}\right)$ be two $\mathcal{F f T S s}$. Then a map $\psi:\left(\mathcal{X}, \mathcal{T}, \mathcal{T}_{f}\right) \rightarrow$ $\left(\mathcal{Y}, \mathcal{S}, \mathcal{S}_{f}\right)$ is

a) Fine fuzzy continuous (in short $F f$ continuous) if $\quad \psi^{-1}(v) \in \quad F f C(X)[F f O(X)] \forall v \in$ $\operatorname{FfC}(\mathcal{Y})[F f O(\mathcal{Y})]$

b) Fine fuzzy spcontinuous ( $F f$ spcontinuous) if $\psi^{-1}(v) \in \quad F f_{\mathfrak{s p} C}(\mathcal{X})\left[F f_{\mathfrak{s p} O} O(X)\right] \forall v \in$ $\operatorname{FfC}(\mathcal{Y})[F f O(\mathcal{Y})]$.

c) Fine fuzzy $\mathfrak{s p}^{*}$ continuous ( $F f \mathfrak{s p}^{*}$ continuous) if $\psi^{-1}(v) \in F f \mathfrak{s p} C(X)[F f \mathfrak{s p} O(X)] \forall v \in$ $F f \mathfrak{s p} C(\mathcal{Y})[F f \mathfrak{s p} O(\mathcal{Y})]$.

d) Fine fuzzy $\mathfrak{s p}^{* *}$ continuous (Ff $\mathfrak{s p}^{* *}$ continuous) if $\quad \psi^{-1}(v) \in$ $F f C(X)[F f O(X)] \forall v \in$ $F f \mathfrak{s p} C(\mathcal{Y})[F f \mathfrak{s p} O(\mathcal{Y})]$

\section{Proposition 5.1}

Every $F f \mathfrak{s p}^{* *}$ continuous is $F f \mathfrak{s p}^{*}$ continuous.

\section{Proof}

Assume two $\mathcal{F} f T S s\left(\mathcal{X}, \mathcal{T}, \mathcal{T}_{f}\right)$ and $\left(\mathcal{Y}, \mathcal{S}, \mathcal{S}_{f}\right)$ A map $\psi:\left(\mathcal{X}, \mathcal{T}, \mathcal{T}_{f}\right) \rightarrow\left(\mathcal{Y}, \mathcal{S}, \mathcal{S}_{f}\right)$ is $F f_{\mathfrak{s p}^{* *} \text { continuous }}$ Then $\forall v \in F f_{\mathfrak{s p}} C(\mathcal{Y}), \psi^{-1}(v) \in F f C(\mathcal{X})$. By Proposition 4.7, every $F f C$ is $F f_{\mathfrak{s p} C .} \therefore \psi^{-1}(v) \in F f \mathfrak{s p} C(\mathcal{X}), \forall v \in$ $F f \mathfrak{s p} C(\mathcal{Y})$. Hence, $\psi$ is $F f \mathfrak{s p}^{*}$ continuous function.

\section{Note 5.1}

The converse of Proposition 5.1 need not be true shows in the following

\section{Example 5.1}

Let $\mathcal{X}=\{\mathrm{a}, \mathrm{b}\}$ be a non empty set. Let $\zeta_{1}, \zeta_{2} \in I^{x}$ be defined by $\zeta_{1}(a)=0.3, \quad \zeta_{1}(b)=0.4 ; \quad \zeta_{2}(a)=0.4$, $\zeta_{2}(b)=0.6$. Let $\mathcal{T}=\left\{0,1, \zeta_{1}, \zeta_{2}\right\}$ be the fuzzy topology on $x$ and $\mathcal{T}_{f}=\left\{0_{\mathrm{X}}, 1_{\mathrm{X}}, \bigcup_{\alpha \in \mathrm{J}}\left\{\mathrm{T}_{\alpha}\right\}\right\}$ be fine fuzzy topology on $\chi$, defined as $\mathcal{T}\left(\zeta_{1}\right)=\mathcal{T}_{1}=\zeta_{1}=$ $\left\{\zeta_{1} \wedge \zeta_{1}=\zeta_{1} \neq 0\right\}, \mathcal{T}\left(\zeta_{2}\right)=\mathcal{T}_{2}=\zeta_{2}=\left\{\zeta_{2} \wedge \zeta_{2}=\right.$ $\left.\zeta_{2} \neq 0\right\}$. Thus, $\left(\mathcal{X}, \mathcal{T}, \mathcal{T}_{f}\right)$ is a $\mathcal{F} f T S$. Let $\psi:(\mathcal{X}, \mathcal{T}$, $\left.\mathcal{T}_{f}\right) \rightarrow\left(\mathcal{X}, \mathcal{T}, \mathcal{T}_{f}\right)$ be an identity map. Then ' $\psi$ ' is $\mathcal{F} f \mathfrak{s p}^{*}$ continuous but not $\mathcal{F} f \mathfrak{s p}^{* *}$ continuous. Since, $\vartheta_{2}(a)=$ $0.5, \vartheta_{2}(b)=0.6 \in F f_{\mathfrak{s p} C}(\mathcal{X})$, but $\psi^{-1}\left(\vartheta_{2}\right)=\vartheta_{2} \notin$

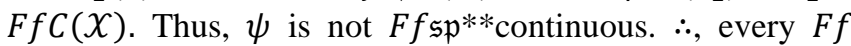
$\mathfrak{s p}^{*}$ continuous need not be $F f \mathfrak{s p}^{* *}$ continuous.

\section{Proposition 5.2}

Every $F f_{\mathfrak{s p}}$ continuous is $F f_{\mathfrak{s p c o n t i n u o u s . ~}}$ 


\section{Proof}

Consider two $\mathcal{F} f$ TSs $\left(\mathcal{X}, \mathcal{T}, \mathcal{T}_{f}\right)$ and $\left(\mathcal{Y}, \mathcal{S}, \mathcal{S}_{f}\right)$. A map $\psi:\left(\mathcal{X}, \mathcal{T}, \mathcal{T}_{f}\right) \rightarrow\left(\mathcal{Y}, \mathcal{S}, \mathcal{S}_{f}\right)$ is fine fuzzy $\mathfrak{s p}^{*}$ continuous. Then, $\quad \forall v \in F f \mathfrak{p p} C(\mathcal{Y}), \quad \psi^{-1}(v) \in$ $F f \mathfrak{s p} C(\mathcal{X})$. By Proposition 4.7, every $F f C$ is $F f_{\mathfrak{s p} C}$. $\therefore$, $\psi^{-1}(v) \in F f_{\mathfrak{s p} C}(\mathcal{X}), \forall v \in F f_{\mathfrak{s p} C}(\mathcal{Y})$. Hence, $\psi$ is $F f \mathfrak{s p c o n t i n u o u s . ~ T h u s , ~ e v e r y ~} F f \mathfrak{s p}^{*}$ continuous is $F f_{\mathfrak{s p c o n t i n u o u s .}}$

\section{Note 5.2}

The converse of Proposition 4.10 need not be true shows in the following

\section{Example 5.2}

Let $\mathcal{X}=\{\mathrm{a}, \mathrm{b}\}$ be a nonempty set. Let $\zeta_{1}, \zeta_{2} \in I^{X}$ be defined by $\zeta_{1}(a)=0.3, \quad \zeta_{1}(b)=0.4 ; \quad \zeta_{2}(a)=0.4$, $\zeta_{2}(b)=0.6$. Let $\mathcal{T}=\left\{0,1, \zeta_{1}, \zeta_{2}\right\}$ be the fuzzy topology on $\mathcal{X}$ and $\mathcal{T}_{f}=\left\{0_{\mathrm{X}}, 1_{\mathrm{X}}, \bigcup_{\alpha \in \mathrm{J}}\left\{\mathrm{T}_{\alpha}\right\}\right\}$ be fine fuzzy topology on $x$, defined as $\mathcal{T}\left(\zeta_{1}\right)=\mathcal{T}_{1}=\zeta_{1}=$ $\left\{\zeta_{1} \wedge \zeta_{1}=\zeta_{1} \neq 0_{\mathrm{X}}\right\}, \mathcal{T}\left(\zeta_{2}\right)=\mathcal{T}_{2}=\zeta_{2}=$ $\left\{\zeta_{2} \wedge \zeta_{2}=\zeta_{2} \neq 0_{\mathrm{X}}\right\}$. Thus, $\left(\mathcal{X}, \mathcal{T}, \mathcal{T}_{f}\right)$ is a $\mathcal{F} f T S$.

Let $\mathcal{Y}=\{\mathrm{c}, \mathrm{d}\}$ be a nonempty set. Let $\delta_{1}, \delta_{2} \in I^{y}$ be defined by $\delta_{1}(a)=0.4, \delta_{1}(b)=0.4 ; \quad \delta_{2}(a)=0.5$, $\delta_{2}(b)=0.6$. Let $S=\left\{0,1, \delta_{1}, \delta_{2}\right\}$ be the fuzzy topology on $\mathrm{Y}$ and $S_{f}=\left\{0_{X}, 1_{X}, \bigcup_{\alpha \in J}\left\{S_{\alpha}\right\}\right\}$ be fine fuzzy topology on $\mathcal{Y}$, defined as $\mathcal{S}\left(\delta_{1}\right)=\mathcal{S}_{1}=\delta_{1}=$ $\left\{\delta_{1} \wedge \delta_{1}=\delta_{1} \neq 0_{\mathrm{X}}\right\}, \mathcal{S}\left(\delta_{2}\right)=\delta_{2}=\delta_{2}=\left\{\delta_{2} \wedge \delta_{2}=\right.$ $\left.\delta_{2} \neq 0_{\mathrm{X}}\right\}$. Now $\left(\mathcal{Y}, \mathcal{S}, \mathcal{S}_{f}\right)$ be $\mathcal{F} f T S$.

Let $\psi:\left(\mathcal{X}, \mathcal{T}, \mathcal{T}_{f}\right) \rightarrow\left(\mathcal{Y}, \mathcal{S}, \mathcal{S}_{f}\right)$ be defined as $\psi(a)=b, \psi(b)=a$. Let $\vartheta \in I^{x}$. Then ' $\psi$ ' is $F f_{\mathfrak{s p c}}$ continuous but not $F f_{\mathfrak{s p}} *$ continuous. $\because, \vartheta(a)=0.6$, $\vartheta(b)=0.3 \in F f_{\mathfrak{s p} C}(\mathcal{Y})$, but $\psi^{-1}(\vartheta)=(0.3,0.6) \notin$ $F f_{\mathfrak{p}} C(\mathcal{X})$. Thus, $\psi$ is not $F f_{\mathfrak{s p}}{ }^{*}$ continuous. Therefore, every $F f \mathfrak{s p c o n t i n u o u s ~ n e e d ~ n o t ~ b e ~} F f \mathfrak{s p}^{*}$ continuous function.

\section{Note 5.3}

Clearly we get the following relations

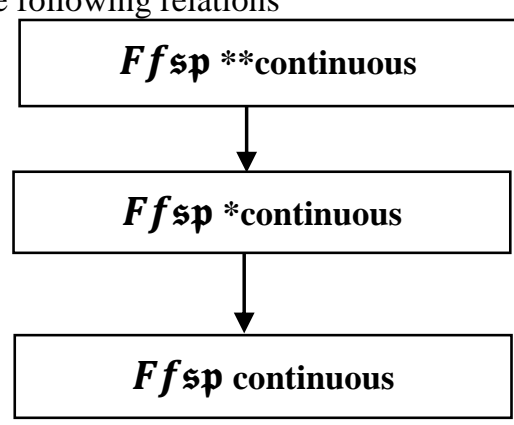

(figure - 4.2)

Relations between $\boldsymbol{F} \boldsymbol{f} \mathfrak{s p c o n t i n u o u s ~ \& ~ o t h e r ~ t y p e s ~ o f ~}$ Ff continuous map

\section{Proposition 5.3}

For a mapping $\psi:\left(\mathcal{X}, \mathcal{T}, \mathcal{T}_{f}\right) \rightarrow\left(\mathcal{Y}, \mathcal{S}, \mathcal{S}_{f}\right)$, the equivalent statements as follows.

(i) $\psi$ is $F f$ spcontinuous.

(ii) $\psi^{-1}(\lambda) \in F f \mathfrak{s p} C(\mathcal{X}), \forall \lambda \in F f C(\mathcal{Y})$.

(iii) $F f \operatorname{Int}\left(F f C l\left(\psi^{-1}(\lambda)\right)\right) \wedge$ $\operatorname{FfCl}\left(F f \operatorname{Int}\left(\psi^{-1}(\lambda)\right)\right) \leq \psi^{-1}(\operatorname{FfCl}(\lambda)) \quad$ for each fine fuzzy set $\lambda$ of $\mathcal{Y}$.

(iv) $\psi(F f \operatorname{Int}(F f C l(v)) \wedge(F f C l(F f \operatorname{Int}(v)))) \leq$ $\operatorname{FfCl}(\psi(v))$ for each fine fuzzy set $v$ of $\mathcal{X}$.

Proof

(i) $\Rightarrow$ (ii)

Let $\lambda \in F f C(\mathcal{Y})$ and $1_{Y}-\lambda \in \operatorname{FfO}(X)$. Hence, $\psi^{-1}\left(1_{Y}-\lambda\right) \in F f_{\mathfrak{s p}} O(\mathcal{X})$. Thus, $\psi^{-1}(\lambda) \in F f_{\mathfrak{s p} O}(\mathcal{X})$.

(ii) $\Rightarrow$ (iii)

Assume that (ii), let fine fuzzy set $\lambda$ of $\mathcal{Y}$, then $\psi^{-1}(F f C l(\lambda))$ is $\mathrm{Ffsp}_{\mathfrak{p}}$ closed in $X$.

$\operatorname{FfInt}\left(F f C l\left(\psi^{-1}(\lambda)\right)\right) \wedge F f C l\left(F f \operatorname{Int}\left(\psi^{-1}(\lambda)\right)\right.$

$$
\begin{aligned}
& \leq F f \operatorname{Int}\left(F f C l\left(\psi^{-1}(F f C l(\lambda))\right)\right) \\
& \wedge F f C l\left(F f \operatorname{Int}\left(\psi^{-1}(F f C l(\lambda))\right)\right) \\
& \leq \psi^{-1}(F f C l(\lambda)) . \\
& \text { (iii) } \Rightarrow \text { (iv) }
\end{aligned}
$$

Let $\mu$ be fine fuzzy set of $\mathcal{X}$, put $\lambda=\psi(\mu)$ then $F f \operatorname{Int}\left(F f C l\left(\psi^{-1}(\psi(\mu))\right)\right) \wedge F f C l\left(F f \operatorname{Int}\left(\psi^{-1}(f(\mu))\right)\right)$

$\leq \psi^{-1}(\operatorname{FfCl}(\psi(\mu)))$ so that, $\operatorname{FfInt}(\operatorname{FfCl}(\mu)) \wedge F f C l(F f \operatorname{Int}((\mu))) \leq$ $\psi^{-1}(F f C l(\psi(\mu)))$, $\psi(\operatorname{FfInt}(F f C l(\mu)) \wedge$

$\operatorname{FfCl}(\operatorname{FfInt}((\mu)))) \leq \operatorname{FfCl}(\psi(\mu))$.

(iv) $\Rightarrow$ (i)

Let $\lambda \in F f O(\mathcal{Y})$. Put $\mu=1_{X}-\lambda$ and $\mu=\psi^{-1}(\lambda)$ then

$$
\begin{aligned}
\psi\left(\operatorname{FfInt}\left(\operatorname{FfCl}\left(\psi^{-1}(\lambda)\right)\right) \wedge\right. & \left.F f C l\left(\operatorname{FfInt}\left(\left(\psi^{-1}(\lambda)\right)\right)\right)\right) \\
& \leq \operatorname{FfCl}\left(\psi\left(\psi^{-1}(\lambda)\right)\right) \\
& \leq \operatorname{FfCl}(\lambda)=\lambda .
\end{aligned}
$$

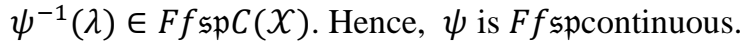

\section{Proposition 5.4}

Let $\left(\mathcal{X}, \mathcal{T}, \mathcal{T}_{f}\right),\left(\mathcal{Y}, \mathcal{S}, \mathcal{S}_{f}\right)$ and $\left(\mathcal{Z}, \mathcal{R}, \mathcal{R}_{f}\right)$ be three $\mathcal{F} f T S s \quad$ and $\quad \psi:\left(\mathcal{X}, \mathcal{T}, \mathcal{T}_{f}\right) \rightarrow\left(\mathcal{Y}, \mathcal{S}, \mathcal{S}_{f}\right) \quad$ and $\phi:$ $\left(\mathcal{Y}, \mathcal{S}, \mathcal{S}_{f}\right) \rightarrow\left(\mathcal{Z}, \mathcal{R}, \mathcal{R}_{f}\right)$ be two maps. Then,

(i) if $\phi \circ \psi$ is $F f_{\text {spopen }}$ and $\psi$ is continuous surjective, then $\phi$ is $F f_{\mathfrak{s p}} O$ map.

(ii) if $\phi \circ \psi$ is $F f O$ and $\phi$ is $F f$ continuous injective, then $\psi$ is $F f_{\mathfrak{s p} O}$ map.

Proof

Let $\eta \in F f O(\mathcal{Y})$. Then, $\psi^{-1}(\eta) \in$ $F f O(X) . \quad \because \phi \circ \psi$ is a $F f \mathfrak{s p} O$ map, then $\quad(\phi \circ \psi)\left(\psi^{-1}(\eta)\right)=$ $\phi\left(\psi\left(\psi^{-1}(\eta)\right)\right)=\phi(\eta) \quad(\because \quad \psi \quad$ is surjec tive) is $\mathrm{a}$ 
fine fuzzy spopen set in $Z$. Thus, $\phi$ is $F f \mathfrak{s p} O$ map.

(ii)

Let $\eta \in F f O(X)$. Then $\phi(\psi(\eta)) \in$ $F f O(Z) . \quad \therefore, \phi^{-1}(\phi(\psi(\eta)))=\psi(\eta)$ ( $\because \phi$ is injective) is a $F f_{\mathfrak{p p} O} O(\mathcal{Y})$. Thus, $\psi$ is $F f_{\mathfrak{s p} O} O$ map.

\section{Proposition 5.5}

Let $\left(\mathcal{X}, \mathcal{T}, \mathcal{T}_{f}\right)$ and $\left(\mathcal{Y}, \mathcal{S}, \mathcal{S}_{f}\right)$ be two $\mathcal{F} f$ TSs and $\psi:\left(\mathcal{X}, \mathcal{T}, \mathcal{T}_{f}\right) \rightarrow\left(\mathcal{Y}, \mathcal{S}, \mathcal{S}_{f}\right)$ be a bijective map. Then the following are equivalent:

(i) $\quad \psi$ is a $F f \mathfrak{s p} O$ map

(ii) $\quad \psi$ is a $F f \mathfrak{s p} C$ map.

(iii) $\quad \psi^{-1}$ is a $F f_{\mathfrak{s p}}$ continuous map.

Proof

(i) $\Rightarrow$ (ii)

Suppose $\zeta \in F f C(X)$. Then $1_{x}-\zeta \in$ $F f O(X)$ and by (i) $\left(1_{x}-\zeta\right) \in F f_{\mathfrak{s p} O}(\mathcal{Y}) . \because \psi$ is bijective, then $\psi\left(1_{x}-\zeta\right)=1_{y}-\psi(\zeta)$. Hence, $\psi(\zeta)$ is $F f_{\mathfrak{s p} C}$ in $\mathcal{Y} . \therefore, \psi$ is a $F f_{\mathfrak{s p} C}$ map.

(ii) $\Rightarrow$ (iii)

Let $\psi$ is a $F f_{\mathfrak{p p} C}$ map and $\zeta \in$ $F f_{\mathfrak{s p} C}(\mathcal{X})$. Since, $\psi$ is bijective, then $\left(\psi^{-1}\right)^{-1}(\zeta)=\psi(\zeta)$ which is a $F f_{\mathfrak{s p} C}$ set in $\mathcal{Y}$. $\therefore$, by Proposition $4.11, \psi^{-1}$ is $F f \mathfrak{s p}$ continuous map.

(iii) $\Rightarrow$ (i)

Let $\delta \in F f O(X)$, by assumption $\psi^{-1}$ is $F f \mathfrak{s p}$ continuous map, then $\left(\psi^{-1}\right)^{-1}(\delta)=\psi(\delta)$ which is a $F f \mathfrak{s p} O$ set in $\mathcal{Y}$. Hence, $\psi$ is a $F f \mathfrak{s p} O$ map.

\section{Proposition 5.6}

Let $\left(\mathcal{X}, \mathcal{T}, \mathcal{T}_{f}\right),\left(\mathcal{Y}, \mathcal{S}, \mathcal{S}_{f}\right)$ and $\left(\mathcal{Z}, \mathcal{R}, \mathcal{R}_{f}\right)$ be three $\mathcal{F} f T S s$. If $\psi:\left(X, T, T_{f}\right) \rightarrow\left(\mathcal{Y}, S, S_{f}\right)$ is $F f \mathfrak{s p}$ continuous map and $\phi:\left(\mathcal{Y}, \mathcal{S}, \mathcal{S}_{f}\right) \rightarrow\left(\mathcal{Z}, \mathcal{R}, \mathcal{R}_{f}\right)$ is $F f$ continuous map then their composition $\phi \circ \psi:\left(\mathcal{X}, \mathcal{T}, \mathcal{T}_{f}\right) \rightarrow$ $\left(Z, \mathcal{R}, \mathcal{R}_{f}\right)$ is also $F f \mathfrak{s p}$ continuous.

\section{Proof}

Let $\zeta$ be any $F f_{\mathfrak{s p}}$ open subset of $\left(Z, \mathcal{R}, \mathcal{R}_{f}\right)$. Then $\left(\phi \circ \psi^{-1}\right)(\zeta)=\left(\psi^{-1} \circ \phi^{-1}\right)(\zeta)=\psi^{-1}\left(\phi^{-1}(\zeta)\right)$. Since, $\phi$ is $F f$ continuous, $\phi^{-1}(\zeta)$ is fine fuzzy open in $\left(Y, S, S_{f}\right)$. Since, $\psi$ is $F f_{\mathfrak{s p}}$ continuous so that $\psi^{-1}\left(\phi^{-1}(\zeta)\right)$ is

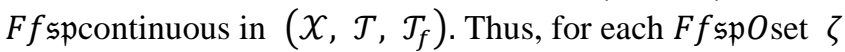
in $\left(Z, \mathcal{R}, \mathcal{R}_{f}\right),(\phi \circ \psi)^{-1}(\zeta) \in F f_{\mathfrak{p}} O(\mathcal{X})$. Hence, $\phi \circ \psi$ is $F f \mathfrak{s p}$ continuous.

\section{CONCLUSION}

The novel kind of space called fine fuzzy topological space is obtained by the notion fine fuzzy quasi coincident has been introduced in this article. Accordingly, the interrelations between fine fuzzy $\mathfrak{s p}$ closed sets with various types of fine fuzzy closed set have been investigated with necessary examples, which revealed that the converse need not be hold are proven with suitable examples. Then, $F f \mathfrak{s p}$ continuous map, $F f \mathfrak{s p}^{*}$ continuous map, $F f \mathfrak{s p}^{* *}$ continuous function and their interrelations have been established. Further, we noticed that its reverse implications are also need not be true. Finally, the fine fuzzy $\mathfrak{s p}$ open, fine fuzzy $\mathfrak{s p}^{*}$ open, fine fuzzy $\mathfrak{s p}^{* *}$ open functions have been defined and its properties were briefly studied. In future, this work will be continued to investigate the fine fuzzy quotient topology, connectedness, disconnectedness and compactness in fine fuzzy bi-topological spaces and their properties.

\section{ACKNOWLEDGEMENT}

I would like to express my sincere thanks to Dr. G. Elaiyaraja for his valuable guidance, constant encouragement and financial support of this research work.

\section{REFERENCES}

1. B. Ahmad, and Athar Kharal, "Fuzzy sets, fuzzy S-open and S-closed mappings", Advances in Fuzzy Systems, Vol. 2009.

2. K. Azad, "On fuzzy semi continuity, fuzzy almost continuity and fuzzy weakly continuity”, Jour. Math. Anal. Appl. 82(1981), 14-32.

3. C. L . Chang, "Fuzzy topological spaces", Jour. Math Anal. Appl. 24(1968), 182-190.

4. S.S. Benchalli and Jenifer J. Karnell, "On fuzzy b-open sets in fuzzy Topological Spaces, J. comp. \& Math. Sci. Vol. 1(2), 127-134(2010).

5. J. Dontchev and M Przemski, "On the various decompositions of continuous and some weakly continuous functions", Acta Mathematica Hun garica, Vol.71, No. 1-2,pp, (1996)109-120.

6. A. Hakeem and Othman, "On fuzzy sp-open sets", Advances in Fuzzy Systems Vol. 2011.

7. N. Levin, "Semi-open sets and semi-continuity in topological spaces", Amer. Math. Monthly, 70 (1963), 36 - 41.

8. O. Njasted, "On some classes of nearly open sets", Pacific J. Math. 15, (1986) 961-970.

9. P. L. Powar and K. Rajak, "Fine irresolute mappings", Journal of Advance Studies in Topology, Vol. 3, No. 4, (2012), 125-139.

10. K. Rajak, "Fine $\boldsymbol{g} \boldsymbol{\delta} \boldsymbol{s}$-separation axioms in fine topological space", International Journal of Advanced Research (2013), Vol. 1, Issue 10, 539-545.

11. K. Rajak, " $\tau^{*} g^{*}$ Semi-closed sets in fine topological spaces". Int Jr. of Mathematical Sciences \& Applications .Vol. 5, 0.2, July-December, (2015).

12. L. A. Zadeh., "Fuzzy sets", Information and control. (1965), 338-353.

\section{AUTHORS PROFILE}

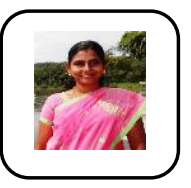

R. Nandhini, M.Sc., M.Phil., is currently doing a doctor of philosophy degree in the Department of Mathematics at Sri Sarada College for Women(Autonomous), Salem, Tamilnadu, India. Then she pursued graduation, master's degree and $\mathbf{M}$. Phil degree in mathematics subject in the same college. She is very much passionate about Mathematics subject and currently working in the area of theory of bipolar fuzzy topology, fuzzy topology, fuzzy dynamical system, and bipolar complex intuitionistic fuzzy graphs. Her research papers have been published in various journals such as International Journal of Mathematical Archive, International Journal of Human Resource Management and Research, Global Journal of Pure and Applied Mathematics, Journal of Applied Science and Computations, The International Journal of Analytical and Experimental Modal Analysis. She has participated and presented her research papers in the international (Five papers) and national conference (Four papers) held at various places. Also, participated in three international and eight national workshops held at various place of Tamil Nadu and also undergone two one month workshop held at Delhi and Kerala respectively. 
D. Amsaveni, M.Sc.,M.Phil,Ph.D is working as an Assistant Professor in Mathematics at Sri Sarada College for Women(Autonomous), Salem, Tamilnadu, India. She has published books and many articles in reputed journals and guiding M.Sc., M.Phil and Ph.D students. She is life member in Ramanujan mathematical society and Reviewer in General Mathematics Notes, International Journal of Mathematics Trends and Technology and Annals of Fuzzy Mathematics and informatics. Her research area includes fuzzy topology and fuzzy modeling. 